\title{
Maximum a posteriori (MAP)-based tag estimation method for dynamic framed-slotted ALOHA (DFSA) in RFID systems
}

\author{
Jinchul Choi and Chaewoo Lee*
}

\begin{abstract}
Radio frequency identification (RFID) is a non-contact technology that uses radio frequency electromagnetic fields to transfer data from a tag attached to an object, for the purposes of automatic identification and tracking. One of the common problems that arise in any RFID deployment is the collision between tags which reduces the efficiency of the RFID system. Dynamic framed-slotted ALOHA (DFSA) is one of the most popular approaches to resolve the tag collision problem. In DFSA, each tag randomly selects one of the time slots of a frame and transmits its data at the slot. Unless the tag successfully transmits its data to a reader, it will try again in the next frame. It is widely known that the optimal performance of framed-slotted ALOHA is achieved when the frame size (i.e., number of time slots) is equal to the number of tags to be identified. So, a reader dynamically adjusts the next frame size according to the number of tags. Thus, it is important to accurately estimate the number of tags. In this article, we propose an accurate maximum a posteriori (MAP)-based tag estimation method with low computational complexity. The idea behind our method is to more accurately determine the most potential number of tags which draws the observed results on the basis of both a posteriori probability and a priori probability. Simulation results show that our method improves the accuracy of tag estimation and the speed of tag identification.
\end{abstract}

\section{Introduction}

Radio frequency identification (RFID) systems that identify tagged objects via near/far-field wireless communications to realize ubiquitous computing are drawing much attention. The operation of RFID systems often involves a situation in which numerous tags are simultaneously placed in the interrogation zone of a single reader. The tags may collide with each other, leading to retransmission of tag data that brings about a waste of bandwidth and an increase in the total delay. To resolve the tag collision problem, a number of tag anti-collision algorithms have been proposed [1]. The primary concern in the algorithms is how to read multiple tags as fast and as reliably as possible.

Tag anti-collision algorithms are mainly grouped into tree-based [2] and ALOHA-based [3] algorithms. Treebased algorithms work by repeatedly splitting the group of colliding tags into two disjoint subsets. The subsets

*Correspondence: cwlee@ajou.ac.kr

Graduate School of Information and Communication, Ajou University, Suwon 443-749, South Korea become smaller and smaller until the number of tags within a subset reduces to one, in which case the tag would be uniquely identified. However, as the number of tags increases, the performance of tree-based algorithms decreases. This is because the colliding tags are successively grouped into two subsets, and each subset may still contain many tags resulting in collisions. The tree-based algorithms have been studied extensively in the literature [4-6].

ALOHA-based algorithms are mostly referred to as Framed-Slotted ALOHA (FSA) [7,8]. In FSA, time is divided into frames of multiple slots and the reader begins its interrogation round by announcing the frame size (i.e., the number of time slots) to the tags. Each tag selects one of the time slots at random and transmits its data at the slot. Unless the tag successfully transmits its data to the reader, it will try again in the next frame. According to [9], the expected throughput $U$ of FSA with $N$ tags and $L$ slots in a frame is given by

$$
U(N, L)=\frac{N}{L}\left(1-\frac{1}{L}\right)^{L-1} .
$$

\section{Springer}

(c) 2012 Choi and Lee; licensee Springer. This is an Open Access article distributed under the terms of the Creative Commons Attribution License (http://creativecommons.org/licenses/by/2.0), which permits unrestricted use, distribution, and reproduction in any medium, provided the original work is properly cited. 
It is obvious from the above equation that the throughput depends on the appropriate choice of frame size $L$, given the number of tags $N$ in the interrogation range. Figure 1 shows the well-known upper bound of the throughput of $e^{-1}$ that is characteristic for slotted ALOHA and also applies to FSA. The maximum throughput occurs when the frame size equals to the number of tags, i.e., $L=N$. Thus, for high performance, it is desirable that the frame size is dynamically adjusted according to the number of unread tags; the mechanism is referred to as Dynamic Framed-Slotted ALOHA (DFSA) [3]. However, DFSA necessitates the reader accurately estimating the number of unread tags to decide an appropriate frame size. To deal with this, various methods to estimate the number of unread tags have been studied in the literature [9-20].

In this article, we propose an accurate and simple maximum a posteriori (MAP)-based tag estimation method for DFSA in RFID systems. In the proposed scheme, we derive a probability mass function (PMF) that describes the relative probability of detection results occurring at a given number of tags and then, based on the derived PMF and the prior tag distribution (if it is postulated), determine the most potential number of tags which draws the detection results observed in a read cycle as the optimal estimate. However, this method may result in a heavy computational load due to the wide search range of tag quantity. To deal with this problem, we propose a simple iterative algorithm based on Newton's method. In our simulations, comparison with several conventional tag estimates shows that the proposed iterative algorithm has lower computational complexity and less error.
The rest of the article is organized as follows. We analyze several important tag estimation methods in the following section. In Section "The proposed tag estimation mechanism", we propose an MAP-based tag estimation with low computational complexity. Simulation results are shown in Section "Numerical results". Finally, we give our concluding remarks in Section "Conclusion".

\section{Related study}

In DFSA, the reader begins its interrogation by first announcing the frame size to all tags within its radio range. Then, each tag randomly selects one of the available time slots and transmits its information at the selected slot. For a given time slot, only three possible outcomes can happen: idle channel, successful transmission, or collision, as shown in Figure 2. The channel is idle if no tag transmits its information at the time slot. A successful transmission means that only one tag sends its information. If two or more tags transmit at the same time slot, the reader suffers from collision and no tag can be read. Based on the detection results, the reader dynamically adjusts the frame size for next read cycle (frame). As mentioned in Introduction section, since the system throughput, which is defined as the ratio between success slots and the frame size, can be maximized when the frame size equals to the number of unread tags, a number of studies have focused on accurate tag estimation [9-20].

The lower bound method [10] is obtained through the observation that a collision involves at least two different tags. Suppose that after carrying out an FSA in which the frame size is set to $F$, the reader can observe $s_{i}$ idle slots, $s_{s}$ success (or singly occupied) slots, and $s_{c}$ collision slots, where $s_{i}+s_{s}+s_{c}=F$. Then, the lower bound method

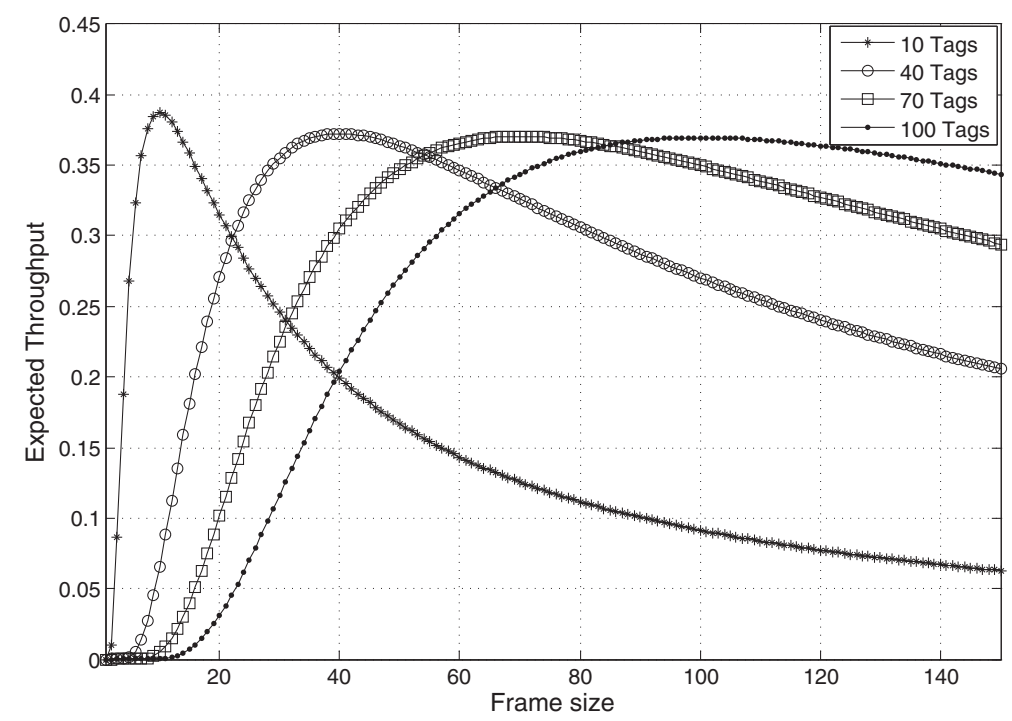

Figure 1 Frame size versus expected throughput in FSA. 


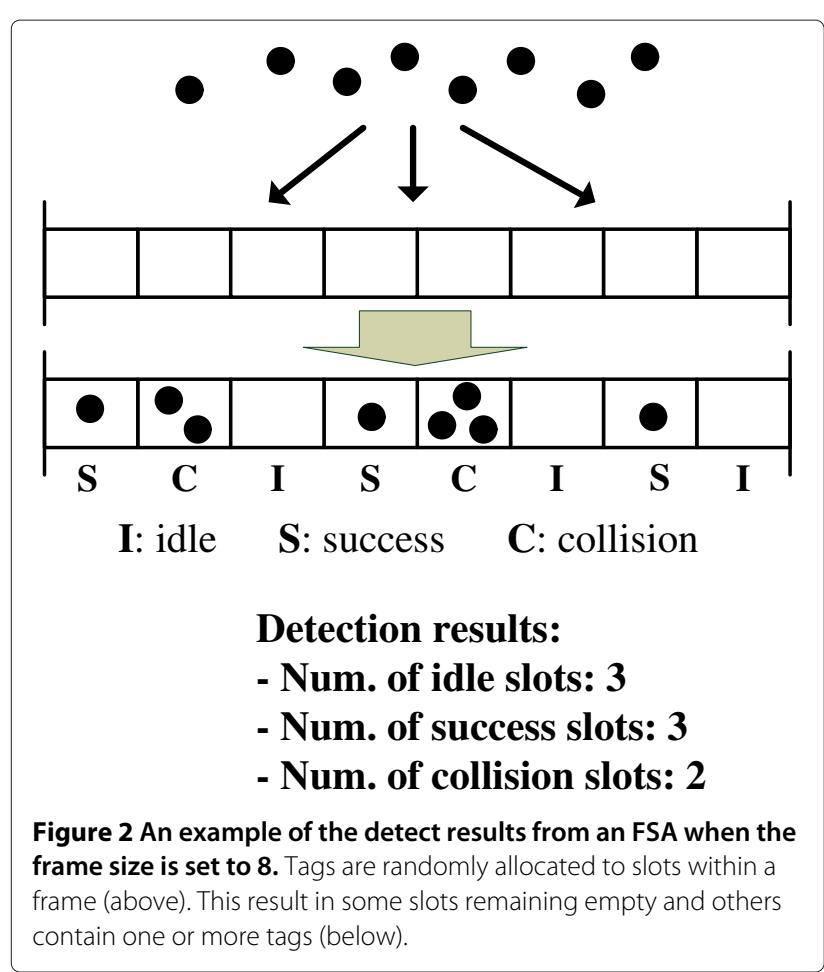

simply estimates the number of tags as $2 s_{c}$. On the other hand, the Schoute method evaluates the expected number of tags per collision slot as 2.39 on the basis of the results in [9]. The authors of [11,12] proposed tag estimation methods based on the Schoute method for RFID anti-collision using FSA. According to these studies, if the frame size is assumed to be the number of tags, the expected value of collision tags is $2.39 s_{c}$. Although the lower bound and the Schoute methods are simple and easy to implement, their assumptions are difficult to confirm in practice. Thus, using the expected value to estimate tag quantity may cause significant error and increase read delay [13].

In [14], the authors proposed tag estimation methods on the basis of approximations to the binomial distribution. Consider $n$ tags are to be read and a frame of $F$ time slots. For a given time slot, the number of tags allocated to the slot is binomially distributed with $n$ Bernoulli trials and $1 / F$ occupied probability. Thus, the probability of finding $r$ tags in the slot is given by

$$
B(r)=\left(\begin{array}{l}
n \\
r
\end{array}\right)\left(\frac{1}{F}\right)^{r}\left(1-\frac{1}{F}\right)^{n-r}
$$

If the frame size $F$ is sufficiently large, (2) can be approximated by a Poisson distribution with mean $n / F$.
Accordingly, the probabilities of idle, successful transmission, and collision for the slot is given by

$$
\begin{aligned}
p_{i} & =B(0)=\left(1-\frac{1}{F}\right)^{n} \approx e^{-\frac{n}{F}} \\
p_{s} & =B(1)=\frac{n}{F}\left(1-\frac{1}{F}\right)^{n-1} \approx \frac{n}{F} e^{-\frac{n}{F}} \\
p_{c} & =1-p_{i}-p_{s}=1-\left(1-\frac{1}{F}\right)^{n}-\frac{n}{F}\left(1-\frac{1}{F}\right)^{n-1} \\
& \approx\left(1-\left(1+\frac{n}{F}\right) e^{-\frac{n}{F}}\right) .
\end{aligned}
$$

From (3a)-(3c), the expectations of $s_{i}, s_{s}$, and $s_{c}$ are given by

$$
\begin{aligned}
E\left[s_{i}\right] & =F\left(1-\frac{1}{F}\right)^{n} \approx F e^{-\frac{n}{F}} \\
E\left[s_{s}\right] & =n\left(1-\frac{1}{F}\right)^{n-1} \approx F \frac{n}{F} e^{-\frac{n}{F}} \\
E\left[s_{c}\right] & =F\left(1-\left(1-\frac{1}{F}\right)^{n}-\frac{n}{F}\left(1-\frac{1}{F}\right)^{n-1}\right) \\
& \approx F\left(1-\left(1+\frac{n}{F}\right) e^{-\frac{n}{F}}\right) .
\end{aligned}
$$

Then, assuming the detection results observed in a read cycle is close to their expectations, the three binomial mean estimators, i.e., zero estimator (ZE), singleton estimator (SE), and collision estimator (CE), can respectively derive their tag estimates $\widehat{n}_{Z E}, \widehat{n}_{S E}$ and $\widehat{n}_{C E}$ by solving the following problems:

$$
\begin{aligned}
e^{-\left(\widehat{n}_{\mathrm{ZE} / F}\right)} & =\frac{s_{i}}{F} \\
\frac{\widehat{n}_{\mathrm{SE}}}{F} e^{-\left(\widehat{n}_{\mathrm{SE} / F}\right)} & =\frac{s_{s}}{F} \\
\left(1-\left(1+\frac{\widehat{n}_{\mathrm{CE}}}{F}\right) e^{-\left(\widehat{n}_{\mathrm{CE} / F}\right)}\right) & =\frac{s_{c}}{F} .
\end{aligned}
$$

In $[15,16]$, the authors proposed the Lottery Frame (LoF) scheme to reduce the multiple-reading by multiple readers with overlapping interrogation regions. LOF adopts the ZE of [14] and dynamically adjusts the system load factor (the ratio of the tag quantity to the frame size) to eliminate the multiple-reading.

In [17], the authors derive the mean values of idle and success slots using a binomial distribution and combine them into a single equation for the number of tags. Solving both (4a) and (4a) for the number of tags $n$, then the Chen method's estimate denoted by $\widehat{n}_{\text {Chen }}$ is given by

$$
\widehat{n}_{\text {Chen }}=(F-1) \frac{E\left[s_{s}\right]}{E\left[s_{i}\right]} \text {. }
$$

Then, assuming that the numbers of idle and success slots observed in a read cycle are identical tot heir expectations, the Chen method estimates the number of tags by substituting the expectations with the observations from 
an FSA. Though the Chen method and the three binomial estimators are simple, some cases (i.e., $s_{i}=0, s_{s}=0$, $s_{c}=0$ ) need special treatment and their accuracies are not satisfactory since the outcomes observed in a read cycle seldom correspond with their expectations [18].

The Vogt method $[19,20]$ uses the distance between the detection result $\left(s_{i}, s_{s}, s_{c}\right)$ and the expected value vector to estimate the number of tags $n$ for which the distance becomes minimal using the exhaustive search. The Vogt method's estimate denoted by $\widehat{n}_{\text {Vogt }}$ is given by

$$
\widehat{n}_{\text {Vogt }}=\min _{n}\left|\left(\begin{array}{c}
E\left[s_{i}\right] \\
E\left[s_{s}\right] \\
E\left[s_{c}\right]
\end{array}\right)-\left(\begin{array}{c}
s_{i} \\
s_{s} \\
s_{c}
\end{array}\right)\right| \text {. }
$$

The Vogt method improves the accuracy of tag estimation compared with the lower bound and the Chen methods. However, the Vogt method requires rather large computational load because it works with no information about the search space.

The Eom method [18] estimates the number of tags by properly determining the number of tags per collision slot. Assuming that the frame size is sufficiently large, it formulates the number of tags per collision slot as two types of equations, one of which consists of the observed detection results (see (8a)) and the other consists of the expected values (see (8b)). Then, it combines them into an iterative algorithm and regards the corresponding convergence as the number of tags per collision slot. The Eom method's two iterative equations are as follows.

$$
\begin{aligned}
& \beta_{k}=\frac{F}{\gamma_{k-1} \cdot s_{c}+s_{s}} \\
& \gamma_{k}=\frac{1-e^{-\frac{1}{\beta_{k}}}}{\beta_{k-1}\left(1-\left(1+\frac{1}{\beta_{k}}\right) e^{-\frac{1}{\beta_{k}}}\right)}
\end{aligned}
$$

where $\beta_{k}$ and $\gamma_{k}$ denote the ratio of the frame size to the tag quantity and the number of tags per collision slot after the $k$ th iteration, respectively. According to [18], the sequence in 8 tends to converge for a reasonable threshold. Then, the Eom method's estimate denoted by $\widehat{n}_{\text {Eom }}$ is given by

$$
\widehat{n}_{\text {Eom }}=\gamma_{k^{*}} \cdot s_{c}+s_{S}
$$

where $\gamma_{k}^{*}$ denote the limit value at the $k^{*}$ th iteration (starting from $\beta_{1}=\infty, \gamma_{1}=2$ ). The Eom method gives a rather accurate estimate when the frame size is large. However, as the frame size decreases, its estimate error increases.

In general, a reader in an RFID system usually reads tags only once in a frame. In this context, Bayesian tag estimate which is available with few observation samples can be an appropriate approach. In [21], Rivest proposes a
Bayesian transmission strategy for an FSA broadcast system. Based on Rivest's work, Floerkemeier also applies the Bayesian transmission strategy to a framed aloha RFID system in $[22,23]$. These works mainly concern about how to minimize the posterior expected value of a risk function (i.e., the posterior expected risk), such as using Bayesian rule to update the probability distribution of tag quantity and adjusting the frame size. However, the tag estimation method has not been discussed in these references.

On the other hand, in [24], using different risk (or loss) functions, the authors propose three Bayesian tag estimation methods: Bayesian mean-square, Bayesian absolute-error, and Bayesian posterior-probability estimation method. According to their simulations, the accuracy of Bayesian posterior-probability estimate (BPE) is the best among their methods. BPE is the same form as the maximum likelihood estimation (MLE) in [13], thus their accuracies are identical. Although BPE and MLE have better estimate performance than other existing methods, there is substantial room for improvement in estimation accuracy because they are only grounded on a posterior probability.

In this article, we propose an accurate MAP-based tag estimation method with low computation complexity. The idea behind our method is to more accurately determine the most potential number of tags which draws the observed results on the basis of both a posteriori probability and a priori probability. Furthermore, we propose a simple iterative algorithm based on Newton's method and reduce computational complexity.

\section{The proposed tag estimation mechanism}

\section{The MAP-based tag estimate}

Consider an RFID system in which the tag anti-collision problem is avoided by the DFSA algorithm. Suppose there is a detection result $s=\left\{s_{i}, s_{s}, s_{c}\right\}$ observed in a read cycle, coming from a distribution with an unknown PMF $f_{0}$ depending on the number of tags. It is however surmised that function $f_{0}$ belongs to a certain family of distributions $\{f(s \mid \theta), \theta \in \Theta\}$, where $\Theta$ denotes the domain of the distributable number of tags, so that $f_{0}=f\left(s \mid \theta_{0}\right)$. The value $\theta_{0}$ is unknown and is referred to as the true value of parameter $\theta$. We want to find an estimate $\hat{\theta}$ which would be as close to the true value $\theta_{0}$ as possible.

Now assume that a distribution $g$ over $\theta$ exists. This allows us to treat $\theta$ as a random variable as in Bayesian statistics. Then, the posterior distribution of $\theta$ is given by

$$
f(\theta \mid s)=\frac{f(s \mid \theta) g(\theta)}{\sum_{i \in \Theta} f(s \mid i) g(i)}
$$

where $g$ is PMF of $\theta$. This is a straightforward application of Bayes' theorem. 
Our method estimates $\theta_{0}$ by finding a value of $\theta$ that maximizes $f(\theta \mid s)$. Thus, the optimal estimate of $\theta$ can be derived as the mode of the posterior distribution of this random variable:

$$
\widehat{\theta}_{\mathrm{MAP}}=\underset{\theta \in \Theta}{\arg \max } \frac{f(s \mid \theta) g(\theta)}{\sum_{i \in \Theta} f(s \mid i) g(i)}
$$

The denominator of the posterior distribution does not depend on $\theta$ and therefore plays no role in the optimization. Consequently, (11) can be simplified as

$$
\widehat{\theta}_{\mathrm{MAP}}=\underset{\theta \in \Theta}{\arg \max } f(s \mid \theta) g(\theta) .
$$

Now we will derive the PMF of $s$ occurring when the number of tags is given by $\theta$, i.e., $f(s \mid \theta)$. All the time slots in a read cycle only belong to one of three possible outcomes such as idle, success, or collision slot. Thus we can conclude that the three outcomes are mutually exclusive and collectively exhaustive (MECE). Then, $f(s \mid \theta)$ is simplified by Bayes' theorem and the property of MECE:

$$
\begin{aligned}
f(s \mid \theta) & =\operatorname{Pr}\left(s_{i}, s_{s}, s_{c} \mid \theta\right) \\
& =\frac{\operatorname{Pr}\left(s_{i}, s_{s}, s_{c}, \theta\right)}{\operatorname{Pr}(\theta)}=\frac{\operatorname{Pr}\left(s_{c} \mid s_{i}, s_{s}, \theta\right) \cdot \operatorname{Pr}\left(s_{i}, s_{s}, \theta\right)}{\operatorname{Pr}(\theta)} \\
& =\frac{\operatorname{Pr}\left(s_{i}, s_{s}, \theta\right)}{\operatorname{Pr}(\theta)}=\operatorname{Pr}\left(s_{i}, s_{s} \mid \theta\right) \\
& =\operatorname{Pr}\left(s_{i} \mid \theta\right) \cdot \operatorname{Pr}\left(s_{s} \mid s_{i}, \theta\right) .
\end{aligned}
$$

Equation (13) holds because $\operatorname{Pr}\left(s_{c} \mid s_{i}, s_{s}, \theta\right)=1$.

Next we need to derive the conditional probability distributions $\operatorname{Pr}\left(s_{i} \mid \theta\right)$ and $\operatorname{Pr}\left(s_{s} \mid s_{i}, \theta\right) . \operatorname{Pr}\left(s_{i} \mid \theta\right)$ means the probability that $\theta$ tags select their respective slots among $F$ slots leaving exactly $s_{i}$ idle slots. Assuming that $\theta=n$, using (2) and (3) we have

$$
\operatorname{Pr}\left(s_{i} \mid \theta\right)=\left(\begin{array}{c}
F \\
s_{i}
\end{array}\right) p_{i}^{s_{i}}\left(1-p_{i}\right)^{F-s_{i}} .
$$

On the other hand, $\operatorname{Pr}\left(s_{S} \mid s_{i}, \theta\right)$ means the probability that $\theta$ tags select their respective slots among $\left(F-s_{i}\right)$ slots with exactly $s_{s}$ slots being singly occupied. Since the $\left(F-s_{i}\right)$ slots belong to either success or collision slots, the probability that one of the slots is singly occupied is $\frac{p_{s}}{p_{s}+p_{c}}$. Thus we have

$$
\begin{aligned}
\operatorname{Pr}\left(s_{s} \mid s_{i}, \theta\right) & =\left(\begin{array}{c}
F-s_{i} \\
s_{s}
\end{array}\right)\left(\frac{p_{s}}{p_{s}+p_{c}}\right)^{s_{s}}\left(1-\frac{p_{s}}{p_{s}+p_{c}}\right)^{\left(F-s_{i}\right)-s_{s}} \\
& =\left(\begin{array}{c}
F-s_{i} \\
s_{s}
\end{array}\right)\left(\frac{p_{s}}{1-p_{i}}\right)^{s_{s}}\left(\frac{p_{c}}{1-p_{i}}\right)^{s_{c}} .
\end{aligned}
$$

(15) holds because $s_{i}+s_{s}+s_{c}=F$ and $p_{i}+p_{s}+p_{c}=1$.
Finally, we have $f(s \mid \theta)$ as follows.

$$
\begin{aligned}
f(s \mid \theta)= & \operatorname{Pr}\left(s_{i} \mid \theta\right) \cdot \operatorname{Pr}\left(s_{s} \mid s_{i}, \theta\right) \\
= & \left(\begin{array}{c}
F \\
s_{i}
\end{array}\right) p_{i}^{s_{i}}\left(1-p_{i}\right)^{F-s_{i}} \cdot\left(\begin{array}{c}
F-s_{i} \\
s_{s}
\end{array}\right) \\
& \times\left(\frac{p_{s}}{1-p_{i}}\right)^{s_{s}}\left(\frac{p_{c}}{1-p_{i}}\right)^{s_{c}} \\
= & \left(\begin{array}{c}
F \\
s_{i}
\end{array}\right)\left(\begin{array}{c}
F-s_{i} \\
s_{s}
\end{array}\right) p_{i}^{s_{i}} p_{s}^{s_{s}} p_{c}^{s_{c}} \\
= & \frac{F !}{s_{i} ! s_{s} ! s_{c} !} p_{i}^{s_{i}} p_{s}^{s_{s}} p_{c}^{s_{c}} .
\end{aligned}
$$

According to (12), using both a prior distribution $g(\theta)$ and a posteriori distribution $f(s \mid \theta)$, we can determine the best estimate $\hat{\theta}$ which is the most potential number of tags. From a statistical point of view, the method of MAP estimation is considered to be more robust (with some exceptions) and yields estimates with good statistical properties [25]. Furthermore, the proposed MAPbased tag estimate is very suitable for estimation under few observation samples [26].

The MAP-based tag estimate requires empirical data about tag detection and a prior distribution over tag quantity $\theta$ to obtain an optimal estimate. A prior distribution is often the purely subjective assessment of an experienced expert. Nevertheless, there are several ways to collect the prior. One reasonable approach is to make the prior a normal distribution with expected value equal to the present tag quantity, with variance equal to the variance of the past several tag quantities. This approach has a property in common with many priors, namely, that the posterior from one problem (present tag quantity) becomes the prior for another problem (next tag quantity) [27]. Other approach is to choose a conjugate prior when they can, to make calculation of the posterior distribution easier. The details of how to collect a prior distribution are shown in [28].

If an informative prior (which expresses specific, definite information about tag quantity) is not postulated or cannot be collected, we use a uniform prior distribution (that is, a constant function). This is based on the principle of indifference, which assigns equal probabilities to all possibilities [29]. Then, the MAP estimate of $\theta$ coincides with MLE in [13] and BPE [24], and thus the accuracy of our estimation method is equal to those of MLE and BPE.

Except for the accuracy of estimation, one obvious concern of both estimates is a tag quantity range over which the maximum probability needs to be searched. If the range is wide, the estimates' computational complexity will be high. To find extremum, our estimate and MLE basically needs to search $N-s_{s}+2 s_{c}$ times in the search range of tag quantity (i.e., from $\theta=s_{s}+2 s_{c}$ to $\theta=N$ ). In this article, we are based on the results of [24], we reduce the computational complexity by narrowing the 
search range. Consequently, our method needs to search $\left|\widehat{\theta}_{M A P}-\bar{\theta}\right|+2$ times where $\bar{\theta}=\ln \left(s_{i} / F\right) / \ln (1-1 / F)$.

On the other hand, if we need to use a uniform prior distribution due to non-informative prior, we can reduce the complexity more. In next section, we propose an iterative algorithm to determine the estimate in less computational complexity when informative prior is not given or a uniform prior distribution is used.

\section{Iterative algorithm to reduce computational complexity}

Let $S$ be a vector of three random variables with a probability distribution with the function $f$ depending on a parameter $x$. Assuming that the prior distribution of $x$ is uniform, the function

$$
\mathcal{L}(x \mid s)=f(s \mid x)=\operatorname{Pr}(S=s \mid x)
$$

considered as a function of $x$, is called the likelihood function (of $x$, given the outcome $s$ of $S$ ) [25]. Let $l(x)$ be a probability distribution with the likelihood function $\mathcal{L}$ depending on a parameter $x$. From (16) and (17), we have

$$
\begin{aligned}
l(x)= & \frac{F !}{s_{i} ! s_{s} ! s_{c} !} p_{i}^{s_{i}} p_{s}^{s_{s}} p_{c}^{s_{c}} \\
= & \frac{F !}{s_{i} ! s_{s} ! s_{c} !}\left(1-\frac{1}{F}\right)^{s_{i} x}\left\{\frac{x}{F}\left(1-\frac{1}{F}\right)^{x-1}\right\}^{s_{s}} \\
& \times\left\{1-\left(1-\frac{1}{F}\right)^{x}-\frac{x}{F}\left(1-\frac{1}{F}\right)^{x-1}\right\}^{s_{c}}
\end{aligned}
$$

where $s_{c} \geq 1$ and $s_{i}+s_{s} \geq 1$. If $s_{c}$ is zero, we can easily conclude that all the tags were read. On the other hand, if both $s_{i}$ and $s_{s}$ are zero, we can conclude that all the tags were collided with each other. As the number of tags increases, the chances of this happening grow higher. The estimate from our method must be the maximum number of tags that the RFID system can read. Thus we only consider a read cycle where $s_{c} \geq 1$ and $s_{i}+s_{s} \geq 1$, hence $x \geq 2$ and $F \geq 2$.

Suppose $\{x \in \mathbb{R} \mid 2 \leq x \leq N\}$ where $N$ is the maximum number of tags that the RFID system can tolerate. It is obvious that $l$ is continuous on the interval because $F \neq 0$. Thus the maximum value is undoubtedly one among $l(2)$, $l(N)$ or the extreme values of $l$. Since the values of $l(2)$ and $l(N)$ can be simply obtained from (18), we focus on the extreme values.

If $l^{\prime}(x)$, i.e., $\frac{\partial}{\partial x} l(x)$, exists, $l$ has a local maximum or minimum at some number $c$ in $[2, N]$ when $l^{\prime}(c)=0$ [30]. To find the first-order derivative of $l$ it is easier to differentiate the logarithm of the function rather than the function itself, hence we first take the natural logarithm on both sides. Then, after implicit differentiation we have

$$
l^{\prime}(x)=l(x) \cdot \frac{\partial}{\partial x} \ln l(x) .
$$

For $l^{\prime}(x)=0, \frac{\partial}{\partial x} \ln l(x)$ should be zero because $l(x)>0$ for all $x$. Thus we will focus on the roots of $\frac{\partial}{\partial x} \ln l(x)$. It is not easy to obtain the roots using elementary algebra, so we apply a numerical approach in finding the roots of general polynomial equation. Let

$$
\begin{aligned}
h(x)= & \frac{\partial}{\partial x} \ln l(x) \\
= & \frac{\partial}{\partial x}\left\{\ln \frac{F !}{s_{i} ! s_{s} ! s_{c} !}+s_{i} x \ln t+s_{s}\left(\ln \frac{x}{F}+(x-1) \ln t\right)\right. \\
& \left.\quad+s_{c} \ln \left(1-t^{x}-\frac{x}{F} t^{x-1}\right)\right\} \\
= & \left(s_{i}+s_{s}\right) \ln t+\frac{s_{s}}{x}-s_{c} \frac{\left(t^{x}+\frac{x}{F} t^{x-1}\right) \ln t+\frac{1}{F} t^{x-1}}{1-\left(t^{x}+\frac{x}{F} t^{x-1}\right)}
\end{aligned}
$$

where $t=1-\frac{1}{F}$. Clearly, $h(x)$ is continuous on the interval $[2, N]$ because $t^{x}+\frac{x}{F} t^{x-1} \neq 1$. Thus we have

$$
\begin{aligned}
h^{\prime}(x)= & -\frac{s_{s}}{x^{2}} \\
& -s_{c} \frac{\left\{\left(t^{x}+\frac{x}{F} t^{x-1}\right) \ln t+\frac{2}{F} t^{x-1}\right\} \ln t+\left(\frac{1}{F} t^{x-1}\right)^{2}}{\left\{1-\left(t^{x}+\frac{x}{F} t^{x-1}\right)\right\}^{2}} .
\end{aligned}
$$

It is also obvious that $h^{\prime}(x)$ is continuous on the interval. Thus, we can use Newton's method which gives better approximations to the roots of a real-valued function [31]. As proved in the Appendix, $h(x)$ has only a single root. Assume that there exists a root $q \in[2, N]$ where $h(q)=0$. According to Newton's method, if $h^{\prime}(q) \neq 0$, the sequence $\left\{q_{k}\right\}_{k=0}^{\infty}$ defined by the iteration

$$
q_{k+1}=q_{k}-\frac{h\left(q_{k}\right)}{h^{\prime}\left(q_{k}\right)}
$$

will converge to $q$ with any initial estimate $q_{0} \in[2, N]$. Nevertheless, it is desirable to choose one as close as possible to the root to reduce the iteration times. To give a better initial estimate, we use the Chen method [17] mentioned in Section "Related study". In addition, the real number of tags is at least $s_{s}+2 s_{c}$. Thus, we set the initial estimate as

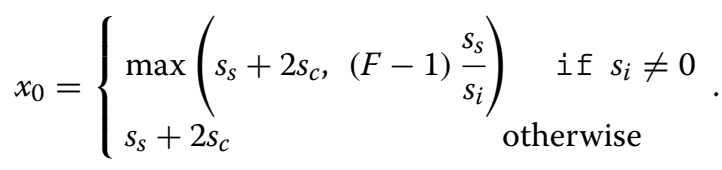

After determining an initial value for $x_{0}$ using (23), we start the iteration based on

$$
x_{k+1}=x_{k}-\frac{h\left(x_{k}\right)}{h^{\prime}\left(x_{k}\right)}
$$


to update tag estimate $x_{k}$ until the difference between $x_{k}$ and $x_{k-1}$ is less than a threshold $\epsilon_{T H}$, i.e.,

$$
\left|x_{k}-x_{k-1}\right|<\epsilon_{T H} .
$$

Let $x^{*}$ be the converged value of $x_{k}$. According to the proof in the Appendix, $h(x)$ is strictly decreasing and has only a single root, i.e., $x^{*}$. Note that $l^{\prime}(x)=l(x) h(x)$ and $l(x)>0$ on the interval $[2, N]$. Since $h(x) \geq 0$ where $x \leq x^{*}$, we can conclude that function $l$ monotonically increases on the interval $\left[2, x^{*}\right]$. On the contrary, $l$ monotonically decreases on the interval $\left[x^{*}, N\right]$ because $h(x) \leq 0$ where $x \geq x^{*}$. Consequently, $l$ attains the maximum value at $x^{*}$. We determine, therefore, the estimate $\hat{\theta}=\left[x^{*}\right]$ that maximizes the likelihood of detection results observed in a read cycle.

\section{Computational complexity analysis}

Among conventional tag estimates, the simpler ones are the lower bound method, the Schoute method, the three binomial mean estimators (i.e., ZE, SE, CE) and the Chen method. Their estimate can be simply obtained. On the other hand, to obtain the value of conditional probability $\operatorname{Pr}(\theta \mid s)$ from $\theta=s_{s}+2 s_{c}$ to $\theta=N$, MLE needs to enumerate the conditional probability $N-s_{s}-2 s_{c}+1$ times. Similarly, the Vogt method needs to search $N-s_{s}-$ $2 s_{c}+1$ times over the search tag range to find extremum. The Eom method uses an iterative algorithm and has less complexity than the Vogt method or MLE. However, a more detailed complexity analysis is likely intractable [18]. Unlike the methods above, by narrowing the search range of tag quantity, BPE reduces its computational complexity. According to [24], BPE needs to search $\left|\widehat{n}_{\mathrm{BPE}}-\bar{n}\right|+2$ where $\widehat{n}_{\mathrm{BPE}}$ denote the Bayesian tag estimate and $\bar{n}=\ln \left(s_{i} / F\right) / \ln (1-1 / F)$.

When we need to use a uniform prior distribution, we can use a simple iterative algorithm based on Newton's method. According to [32], using Newton's method, the computational complexity of calculating a root of a function $a(x)$ with $c$-digit precision, provided that a good initial approximation is known, is $\mathrm{O}((\log c) F(c))$ where $F(c)$ is the cost of calculating $a(x) / a^{\prime}(x)$ with $c$-digit precision. On the other hand, when an informative prior distribution is postulated, the complexity of our method is similar to that of BPE. The computational complexity of the primary estimates is listed in Table 1.

\section{Numerical results}

Computer simulations are performed to check and extend the analytical results of the previous section. We consider a single reader and a number of tags under error-free channel environment. The prior distribution for tag quantity is assumed a normal distribution with mean $\mu$ and variance $\sigma^{2}$. We set the frame size and the tag quantity of
Table 1 Computational complexity

\begin{tabular}{ll}
\hline Estimation method & Computational complexity \\
\hline The Vogt method & $O\left(N-s_{S}-2 s_{C}+1\right)$ \\
MLE & $O\left(N-s_{S}-2 s_{C}+1\right)$ \\
BPE & $O\left(\left|\widehat{n}_{B P E}-\bar{n}\right|+2\right)$ \\
MAP (with a uniform prior) & $O((\mid \log C) F(c))$ \\
MAP (with a non-uniform prior) & $O\left(\left|\widehat{n}_{M A P}-\bar{n}\right|+2\right)$ \\
\hline
\end{tabular}

each read cycle to generate from the prior normal distribution and obtain detection result $S$ from the read cycle. Then, we perform the proposed tag estimation method. Finally, we obtain the average estimate after completing 1,000 cycles. In our method, $\epsilon_{\mathrm{TH}}$ is set to 0.001 .

Figures 3 and 4 show the average estimation error which is defined as the average difference between the real number and the estimated number of tags. For the simulation, we set the frame size to 128 or 32 and vary the mean $\mu$ from 8 to 256 or from 2 to 64 , respectively. The variance $\sigma^{2}$ is set to $0.05 \mu$ or $0.1 \mu$, respectively. Note that when a uniform prior distribution is considered, our method is actually MLE [13] and has the same results as MLE and BPE. Thus, we do not give the estimation results of our method which incorporates a uniform prior distribution in our simulations. In addition, the performance of SE [14] is insufficient and we do not give its results.

When the number of tags is considerably less than the frame size, Lower bound method is the most efficient among the methods. However, as the number of tags increases, its estimation errors increase steeply. Among the existing methods, BPE and MLE which have the similar curves to the Eom method show reasonable performance. On the other hand, the accuracies of other estimates such as ZE, CE, Chen, Schoute and Vogt methods are not satisfactory. Figures 3 and 4 present that the proposed method provides more precise estimation than the other methods except the region in which the number of tags is comparatively less than the frame size.

Figure 5 shows the simulation results for estimate computational complexity, where the computational complexity can also be seen in Table 1 . For the simulation, we set the frame size to 128 or 32 . If a uniform prior distribution is postulated, our method can reduce the computational complexity using the iterative algorithm in (24). The computational complexity is defined as the average number of iterations performed to obtain the tag estimate. Note that the complexity of MLE and the Vogt estimates depend on the maximum number of tags $N$ and are quite high (i.e., more than $N / 2$ ). In addition, the accuracies of simple estimates such as the lower bound method, the Schoute method, the three binomial mean estimators and the Chen method are insufficient. Thus, we do not give the results of them in Figure 5. Figure 5 presents that the complexity 


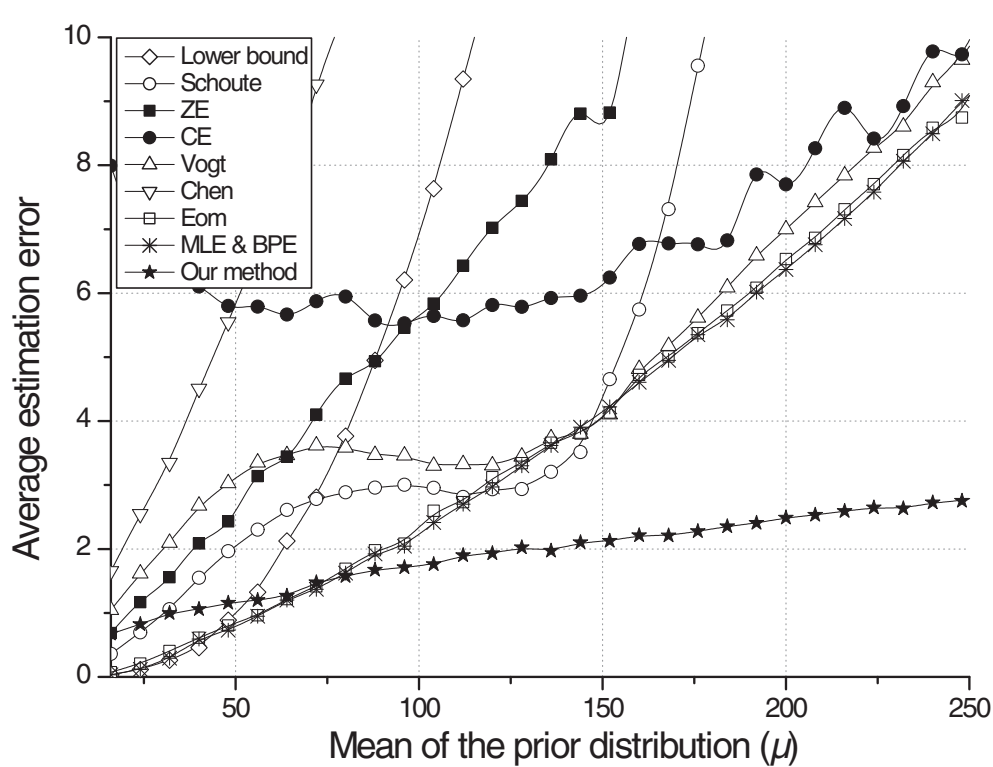

Figure 3 Difference between the real number and the estimated number of tags when the frame size is set to 128 where $\sigma^{2}=0.05 \mu$.

of our iterative algorithm is less than that of BPE. When a non-uniform prior distribution is postulated, the complexity of our method is a little bit high than that of BPE. Nevertheless, our method gives more accurate estimate than BPE and MLE as shown in Figures 3 and 4.

To evaluate the performance of DFSA in which each tag estimation method is employed, we measure the number of time slots used to detect all the tags when the next frame size is set to the number of unread tags estimated by each method. In our method, the mean value for the next prior distribution is adjusted according to its current estimate. An initial frame size and the minimum size are respectively set to 64 and 8 , and the mean $\mu$ varies from 100 to 1000. In Figure 6, we compare the RFID system efficiency, which is defined as the ratio of the number of success slots to the number of slots used to detect all

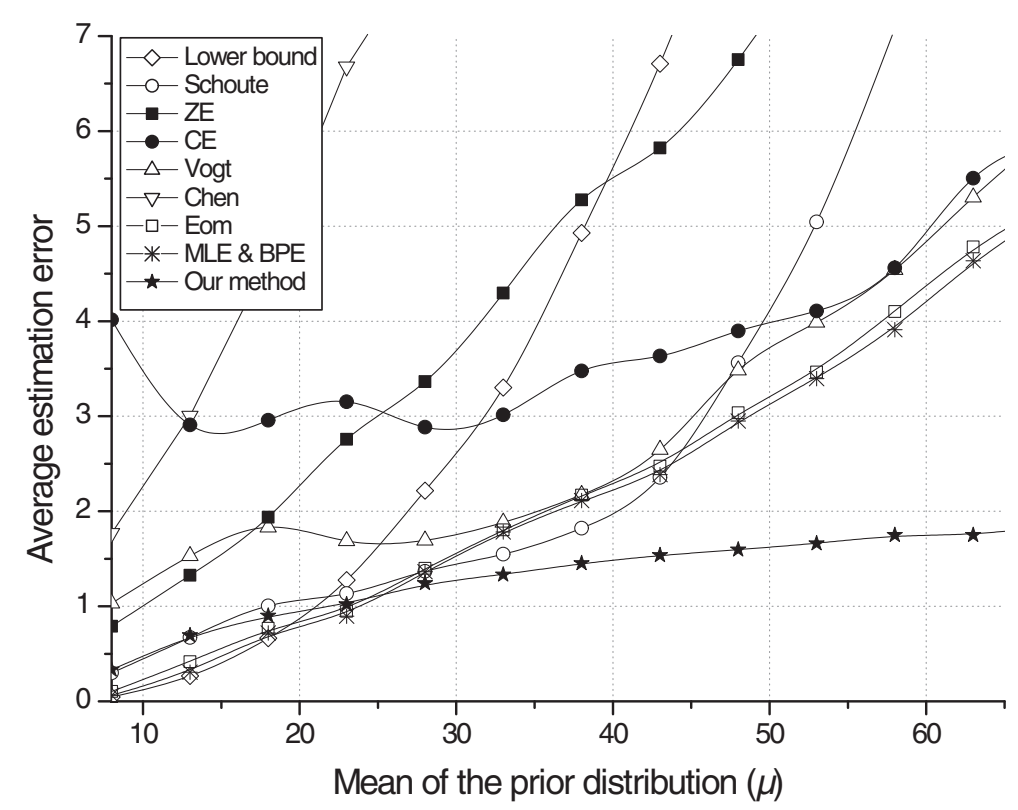

Figure 4 Difference between the real number and the estimated number of tags when the frame size is set to 32 where $\sigma^{2}=0.1 \mu$.

Figures 3 and 4 show the mean estimation error which is defined as the mean difference between the real number and the estimated number of tags. 


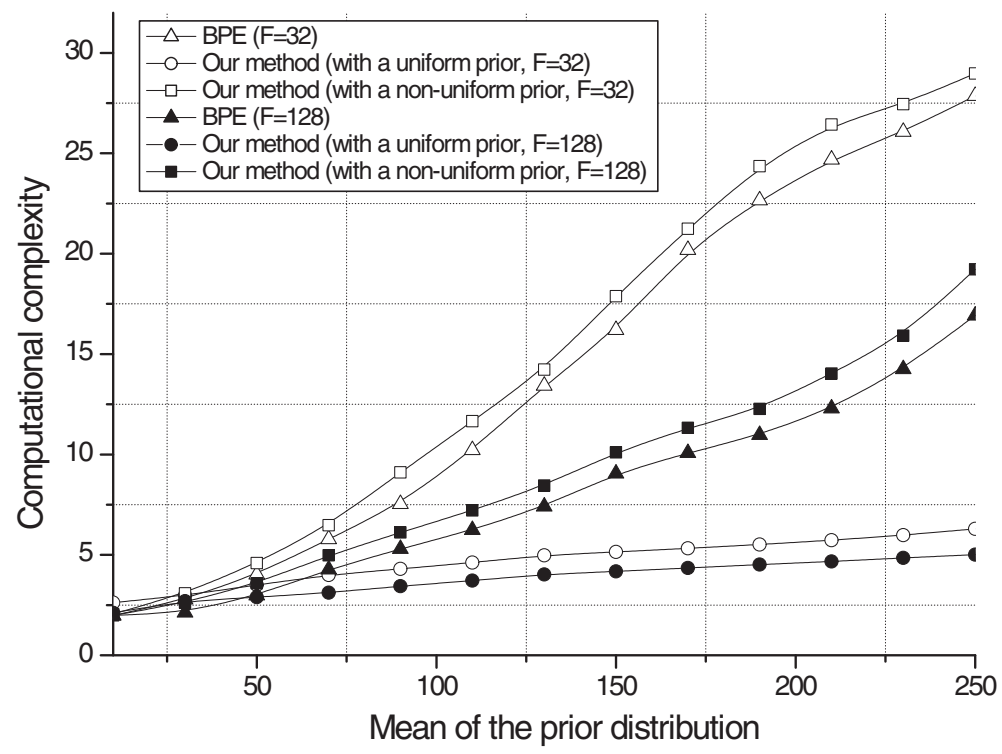

Figure 5 Simulation results for estimate computational complexity where $\sigma^{2}=0.1 \mu$.

the tags. Figure 6 shows that our tag estimation method gives the highest system efficiency compared to the others. It also demonstrates that the performance of DFSA can be nearly improved to the maximum efficiency of Slotted ALOHA (about 36.8\%) using our tag estimation method.

\section{Conclusion}

In this article, we have proposed an accurate MAP-based tag estimation method for DFSA in RFID systems and improved the proposed method to reduce computational complexity. The proposed method can be used to obtain a point estimate of an unobserved tag quantity on the basis of empirical detection data and an informative prior. From our theoretical derivation and simulation results, we draw the conclusions as follows.

The proposed MAP-based tag estimation improves the accuracy of tag estimation and requires less time slots to read out all tags than the other existing methods. When

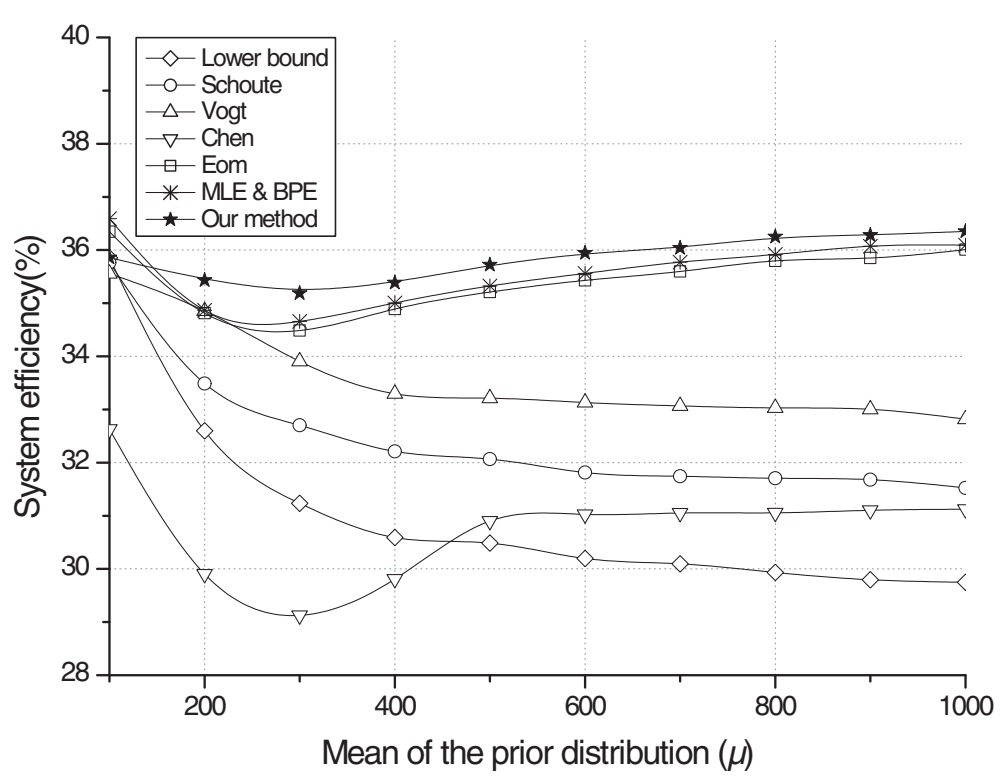

Figure $\mathbf{6}$ System efficiency of DFSA when the frame size is set to the estimated number of tags where $\sigma^{2}=\mathbf{0 . 1} \boldsymbol{\mu}$. Also shows the corresponding system efficiency, which is defined as the ratio of the number of success slots to the number of slots used to detect all the tags. 
an informative prior is not postulated or a uniform prior is used, our method can reduce computational complexity using the proposed iterative algorithm. Nevertheless, our method produces the same estimates as MLE and BPE. When the precise number of tags is not given, which is usual in practice, our method can achieve accurate estimation performance. Therefore, our method can become a suitable candidate for such a mechanism and can be useful for engineers to implement the DFSA algorithm in a real RFID system.

\section{Appendix}

\section{Proof that $h(x)$ has only a single root}

The function $h$ is continuous on the closed interval $[2, N]$. According to the intermediate value theorem [30], if $h(2) h(N)<0$ and $h$ is a strictly monotonic function, $h$ has only a single root in the interval. To prove that $h$ has only a single root, we show that $h(2)>0, h(N)<0$, and $h^{\prime}(x)<0$. Note that we only consider a read cycle where $s_{c} \geq 1, s_{i}+s_{s} \geq 1$, hence $F \geq 2$.

The proof consists of three steps. In the first step, we prove using contradiction that $h(2)>0$. Let

$$
t=1-\frac{1}{F}
$$

From (11), we have

$$
\begin{aligned}
h(2) & =\left(s_{i}+s_{s}\right) \ln t+\frac{s_{s}}{2}-s_{c} \frac{\left(t^{2}+\frac{2}{F} t\right) \ln t+\frac{1}{F} t}{1-\left(t^{2}+\frac{2}{F} t\right)} \\
& =\left(F-s_{c}\right) \ln t+\frac{s_{s}}{2}-s_{c}\left\{\left(F^{2}-1\right) \ln t+(F-1)\right\} \\
& =F\left(1-s_{c} F\right) \ln t+\frac{s_{s}}{2}-s_{c}(F-1) .
\end{aligned}
$$

Suppose that $h(2) \leq 0$. Then, we have

$$
F\left(1-s_{c} F\right) \ln t+\frac{s_{s}}{2}-s_{c}(F-1) \leq 0
$$

We subtract $\frac{s_{s}}{2}-s_{c}(F-1)$ from each side of the inequality and then divide both sides by $s_{c}(F-1)$. Then, we have

$$
-\left(F-\frac{1}{s_{c}}\right)\left(\frac{F}{F-1}\right) \ln t \leq 1-\frac{s_{s}}{2 s_{c}(F-1)}
$$

The right-hand side of (28) is at most one because $\frac{s_{S}}{2 s_{c}(F-1)} \geq 0$. If the left-hand side of (28) is larger than one, it can be concluded that inequality (28) is invalid. Now we show that the left-hand side of (28) is larger than one. Given any $F$, the left-hand side of (28) is minimized when $s_{c}=1$ because $\ln t<0$. Substituting $s_{c}$ with one, the left-hand side of (28) is given by $-F \ln t$. Let

$$
\begin{aligned}
m(F) & =-F \ln t \\
& =-F \ln \left(1-\frac{1}{F}\right)=\ln \left(1+\frac{1}{F-1}\right)^{F} .
\end{aligned}
$$

$m(F)$ has following properties.

(i) $m(2)>1$

(ii) $\lim _{F \rightarrow \infty} m(F)=1$ because $\lim _{F \rightarrow \infty}\left(1+\frac{1}{F-1}\right)^{F}=e$.

(iii) Assuming $m$ is a continuous function, we have $m^{\prime}(F)=-\left(\ln \left(1-\frac{1}{F}\right)+\frac{1}{F-1}\right)$. Since $m^{\prime}(F)<0$ where $F \geq 2, m(F)$ is strictly decreasing. Since frame size $F$ is finite in practice, taken the three properties together, we have $m(F)>1$. This contradicts the proposition that $h(2) \leq 0$. Therefore, it can be concluded that $h(2)>0$ where $s_{c} \geq 1$.

In the second step, we show that $h(N)<0 . h(N)$ is given by

$h(N)=\left(s_{i}+s_{s}\right) \ln t+\frac{s_{s}}{N}-s_{c} \frac{\left(t^{N}+\frac{N}{F} t^{N-1}\right) \ln t+\frac{1}{F} t^{N-1}}{1-\left(t^{N}+\frac{N}{F} t^{N-1}\right)}$.

Assuming that $N$ is sufficiently large, both $t^{N}$ and $\frac{N}{F} t^{N-1}$ can be approximated to zero. Thus, we have

$$
\lim _{N \rightarrow \infty} h(N)=\left(s_{i}+s_{s}\right) \ln t
$$

Since $\ln t<0$ and $s_{i}+s_{s} \geq 1$, it can be concluded that $h(N)<0$ where $N$ is sufficiently large.

In the final step, we prove that $h^{\prime}(x)<0$ on the interval $[2, N]$. From (21) we have

$$
\begin{aligned}
h^{\prime}(x)= & -\frac{s_{s}}{x^{2}} \\
& -s_{c} \frac{\left\{\left(t^{x}+\frac{x}{F} t^{x-1}\right) \ln t+\frac{2}{F} t^{x-1}\right\} \ln t+\left(\frac{1}{F} t^{x-1}\right)^{2}}{\left\{1-\left(t^{x}+\frac{x}{F} t^{x-1}\right)\right\}^{2}} \\
= & -\frac{s_{s}}{x^{2}}-\frac{s_{c}}{F} t^{x-1} \frac{n(x)}{\left\{1-\left(t^{x}+\frac{x}{F} t^{x-1}\right)\right\}^{2}} .
\end{aligned}
$$

where $n(x)=\{(F-1+x) \ln t+2\} \ln t+\frac{1}{F} t^{x-1}$. Since both $\frac{s_{c}}{F} t^{x-1}$ and $\left\{1-\left(t^{x}+\frac{x}{F} t^{x-1}\right)\right\}^{2}$ are larger than zero, if $n(x)$ is larger than or equal to zero, then $h^{\prime}(x)$ is negative at all $x$. Now we prove that $n(x) \geq 0$ by showing that $n(x)$ is strictly increasing and $n(2) \geq 0$. It is obvious that $n(x)$ is continuous on the interval. Thus we have

$$
\begin{aligned}
n^{\prime}(x) & =\ln ^{2} t+\frac{1}{F} t^{x-1} \ln t \\
& =\frac{1}{F} \ln t\left(F \ln t+t^{x-1}\right) .
\end{aligned}
$$


In the first step, we have proved that $-F \ln t>1$, thus we have $F \ln t<-1$. Since $t^{x-1}<1, F \ln t+t^{x-1}$ is less than zero. It can, therefore, be concluded that $n^{\prime}(x)>0$, i.e., $n(x)$ is strictly increasing. Next we show that $n(2)$ is positive at all $F$. Let $x$ be two and $F$ be the function $n$ 's variable and allowed to vary freely. Let $r(F)$ be a function that describes the value of $n(2)$ at varying $F$. Then, we have

$$
r(F)=\{(F+1) \ln t+2\} \ln t+\frac{1}{F} t .
$$

$r(F)$ has following properties.

(i) $r(2)>0$.

(ii) $\lim _{F \rightarrow \infty} r(F)=0$.

(iii) Assuming $r$ is a continuous function, we have $r^{\prime}(F)=\frac{2}{F(F-1)}\left\{F \ln t+1+\ln t+\frac{1}{2 F} t\right\}$. The derivation of $r^{\prime}(F)$ is as follows. Since $t=1-\frac{1}{F}$, $\frac{\partial t}{\partial F}=\frac{1}{F^{2}}$. Thus, we have $\frac{\partial r(F)}{\partial F}=\frac{1}{F^{2}} \frac{\partial r(F)}{\partial t}$. Then, $r^{\prime}(F)$ is given by

$$
\begin{aligned}
r^{\prime}(F) & =\frac{1}{F^{2}} \frac{\partial r(F)}{\partial t} \\
& =\frac{1}{F^{2}}\left\{\frac{F+1}{t} \ln t+\frac{(F+1) \ln t+2}{t}+\frac{1}{F}\right\} \\
& =\frac{1}{F^{2}} \frac{1}{t}\left\{(F+1) \ln t+(F+1) \ln t+2+\frac{1}{F} t\right\} \\
& =\frac{2}{F(F-1)}\left\{(F+1) \ln t+1+\frac{1}{2 F} t\right\} \\
& =\frac{2}{F(F-1)}\left\{F \ln t+1+\ln t+\frac{1}{2 F} t\right\} .
\end{aligned}
$$

From $F \ln t<-1$, we have $F \ln t+1<0$. This inequality can be transformed into $\ln t<-\frac{1}{F}$. Since $\frac{t}{2}<1$, we have $\ln t<-\frac{1}{F}<-\frac{1}{2 F} t$. Thus $r^{\prime}(F)$ is negative for all $F$. Taken three properties together, it can be concluded that $r(F)>0$, i.e., $n(2)>0$. Consequently, we have that $n(x)>0$. Therefore, we can conclude that $h^{\prime}(x)<0$ on the interval $[2, N]$.

From the preceding three steps, it can be concluded that $h(x)$ has only a single root in the interval $[2, N]$ where $s_{c} \geq 1$ and $s_{i}+s_{s} \geq 1$.

\section{Competing interests}

The authors declare that they have no competing interests.

\section{Acknowledgements}

This research was supported by the Basic Science Research Program through the National Research Foundation of Korea (NRF) funded by the Ministry of Education, Science and Technology (2012R1A1A2007605).
Received: 5 March 2012 Accepted: 17 July 2012

Published: 23 August 2012

\section{References}

1. D Shih, P Sun, DC Yen, S Huang, Taxonomy and survey of RFID anti-collision protocols. Comput. Commun. 29(11), 2150-2166 (2006)

2. DR Hush, C Wood, in Proc. of IEEE Int. Symp. Information Theory. Analysis of tree algorithm for RFID arbitration, (1998), pp. 107-107

3. S Lee, S Joo, C Lee, in Proc. of Mobiquitous. An enhanced dynamic framed slotted aloha algorithm for RFID tag identification, (2005) pp. 98-113

4. C Law, K Lee, KY Siu, in Proc. of Mobicom. Efficient memoryless protocol for tag identification, (2000), pp. 75-84

5. M Nanjundaiah, V Chaudhary, in Proc. of AINA. Improvement to the protocol specification for $900 \mathrm{MHz}$ class 0 radio frequency identification tag. vol. 2, 2005, pp. 616-620

6. J Myung, W Lee, J Srivastava, Adaptive binary splitting for efficient RFID tag anti-collision. IEEE Commun. Lett. 10(3), 144-146 (2006)

7. EPCglobal Inc., Radio-frequency Identification Protocols Class-1 Generation-2 UHF RFID Protocol for Communications at $860 \mathrm{MHz}-960 \mathrm{MHz}$ Ver. 1.0.9 (2005). Available at http://www.epcglobalinc.org/ standards_technology/specifications.html

8. ISO/IEC 18000-6, Parameters for Air Interface Communications at 860 $\mathrm{MHz}$ to $960 \mathrm{MHz}$ Information Technology-Radio Frequency Identification for Item Management, ISO/IEC 18000-6, Part 6 (2004). Available at http:// www.iso.org/iso/home/store/catalogue_tc/catalogue_detail.htm? csnumber $=46149$

9. FC Schoute, Dynamic frame length ALOHA. IEEE Trans. Commun. 31(4), 565-568 (1983)

10. Z Wang, D Liu, X Zhou, X Tan, J Wang, H Min, Anti-collision Scheme Analysis of RFID System. Auto-ID Labs White Paper (2007). Available at http://www.autoidlabs.org/single-view/dir/article/6/281/page.html

11. J Cha, J Kim, in Proc. of ICPADS, Novel anti-collision algorithms for fast object identification in RFID system. vol. 2, 2005, pp. 63-67

12. C Floerkemeier, in Proc. of PerCom. Transmission control scheme for fast RFID object identification, (2006), pp. 457-462

13. W Chen, An accurate tag estimate method for improving the performance of an RFID anticollision algorithm based on dynamic frame length ALOHA. IEEE Trans. Autom. Sci. Eng. 6(1), 9-15 (2009)

14. M Kodialam, T Nandagopal, in Proc. of Mobicom. Fast and Reliable Estimation Schemes in RFID Systems, (2006) pp. 322-333

15. C Qian, H Ngan, Y Liu, in Proc. of PERCOM. Cardinality estimation for large-scale RFID systems, (2008), pp. 30-39

16. C Qian, H Ngan, Y Liu, L Ni, Cardinality estimation for large-scale RFID systems. IEEE Trans. Parallel Distrib. Syst. 22(9), 1441-1454 (2011)

17. W Chen, G Lin, An efficient anti-collision method for tag identification in a RFID system. IEICE Trans Commun. E89-B(12), 3389-3392 (2006)

18. J Eom, T Lee, Accurate tag estimation for dynamic framed-slotted ALOHA in RFID systems. IEEE Commun. Lett. 14(1), 60-62 (2010)

19. H Vogt, in Proc. of ICSMSC. Multiple object identification with passive RFID tags. vol. 3, 2002, pp. 6-9

20. H Vogt, in Proc. of ICPC. Efficient object identification with passive RFID tags, (2002), pp. 98-113

21. R Rivest, Network control by bayesian broadcast. IEEE Trans. Inf. Theory. 33(3), 323-328 (1987)

22. C Floerkemeier, in Proc. of IEEE PerCom. Transmission control scheme for fast RFID object identification, (2006), pp. 1-6

23. C Floerkemeier, in Proc. of IEEE RFID. Bayesian transmission strategy for framed ALOHA based RFID protocols, (2007), pp. 228-235

24. HWu, Y Zeng, Bayesian tag estimate optimal frame length for anti-collision Aloha RFID system. IEEE Trans. Autom. Sci. Eng. 7(4), 963-969 (2010)

25. JM Mendel, Lessons in Estimation Theory for Signal Processing, Communications, and Control. (Prentice Hall, New York, 1995)

26. JO Berger, Statistical Decision Theory and Bayesian Analysis. (Springer, New York, 1985)

27. J Berger, J Bernardo, D Sun, The formal definition of reference priors, Annal. Stat. 37(2), 905-938 (2009)

28. K Murphy, Conjugate Bayesian analysis of the Gaussian distribution Technical report UBC (2007). Available at www.cs.ubc.ca/murphyk/ Papers/bayesGauss.pdf 
29. E Jaynes, Probability Theory: The Logic of Science. (Cambridge University Press, Cambridge, MA, 2003)

30. HJ Keisler, Elementary Calculus: An Infinitesimal Approach, 2nd ed. (Prindle Weber \& Schmidt, Boston, 1986)

31. P Deuflhard, Newton Methods for Nonlinear Problems: Affine Invariance and Adaptive Algorithms, 1st ed. (Springer Press, New York, 2004)

32. J Borwein, P Borwein, Pi and the AGM: A Study in Analytic Number Theory and Computational Complexity. (Wiley Interscience, 1987)

doi:10.1186/1687-1499-2012-268

Cite this article as: Choi and Lee: Maximum a posteriori (MAP)-based tag estimation method for dynamic framed-slotted ALOHA (DFSA) in RFID systems. EURASIP Journal on Wireless Communications and Networking 2012 2012:268.

\section{Submit your manuscript to a SpringerOpen ${ }^{\mathcal{O}}$ journal and benefit from:}

- Convenient online submission

- Rigorous peer review

- Immediate publication on acceptance

- Open access: articles freely available online

- High visibility within the field

- Retaining the copyright to your article

Submit your next manuscript at $\gg$ springeropen.com 